\title{
Histogenesis of suprarenal glands at different gestational age groups
}

\author{
Ravindra Kumar Boddeti', Subhadra Devi Velichety ${ }^{2}$ \\ ${ }^{1}$ Lecturer, ${ }^{2}$ Professor and Head, Department of Anatomy, Sri Padmavathi Medical College for Women, Sri \\ Venkateswara Institute of Medical Sciences, SVIMS University, Tirupathi, Andhra Pradesh, India
}

Background: The human foetal suprarenal gland is structurally variant from its adult counterpart. The most distinctive features of human foetal suprarenal gland and histologically unique foetal zone, was described first by Elliott and Armour in 1911. After the first trimester, the centrally located foetal zone accounts for most of the foetal adrenal mass. The outer zone of the foetal suprarenal gland is called the "definitive zone or neo cortex"; this zone likely gives rise to the adult adrenal glomerulosa. A third zone called "transitional zone", lies just between the neocortex and foetal zone and is believed to develop into the zona fasciculata. Aims and Objectives: The current study was designed to study the histogenesis of suprarenal glands at different gestational age groups. Materials and Methods: Twenty-eight formalin preserved dead embryos and foetuses of both sexes, were obtained from the Govt. Maternity Hospital \& S.V.Medical College, Tirupati, Andhra Pradesh, India. Specimens were grouped according to their gestational age groups $(A, B, C, D) A=0-12$ weeks, $B=13-24$ weeks, $C=25-36$ weeks and $D=$ more than 36 weeks of gestation. Specimens from group $A$ were subjected to serial section as this group consists of embryos, and other groups were sectioned coronal and subjected to routine histological processing for H\&E staining. Sections were observed for cellular details under light microscopy with 10X and 40X magnifications, and the same were photographed by microphotography. Results: Based upon the gestational age groups, histogenesis of the suprarenal gland was observed and correlated with the available literature, and the detailed results, discussion will be dealt at the time of discussion. Conclusions: Histological observation of the all the specimens observed in the present study are in agreement with those reported in the literature except that they appeared earlier in the present study than that reported in the literature. Capsule of suprarenal gland appeared at 12 weeks, sympatho-chromaffin bundles appeared before 6 weeks and zonation of cortex was observed at 8 weeks in the present study when compared to the time of appearance reported in the literature as 14 weeks, after 6 weeks and after 12 weeks respectively in the literature.

Key Words: Adrenal gland; Gestational age; Gonadal ridge; Suprarenal ridge;

Sympatho-chromaffin cells; Foetal zone; Primitive cortex

Access this article online

Website:

http://nepjol.info/index.php/AJMS

DOI: 10.3126/ajms.v10i3.22820

E-ISSN: 2091-0576

P-ISSN: 2467-9100

\section{INTRODUCTION}

The endocrine system is a collection of glands that works in parallel with the nervous system and secretes chemical substances called hormones. Hormones control growth and maturation and maintain homeostasis. Animals with well-developed nervous and circulatory systems have an endocrine system. Endocrine systems of crustaceans, arthropods, and vertebrates show similarities. The vertebrate endocrine system consists of glands (pituitary, thyroid, adrenal), and diffuse cell groups scattered in

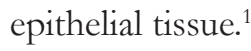

Endocrine glands develop from all three basic germ layers i.e., ectoderm, endoderm and mesoderm. The type of endocrine production depends on its developmental 
origin from the germ layer. Glands of ectodermal and endodermal origin produce peptide and amine hormones. Glands of mesodermal origin secrete lipid hormones. Prenatal development of endocrine glands demonstrates specific time pattern in growth and differentiation. These provide explanation for association between glandular differentiation and concomitant changes in particular target tissues. ${ }^{1,2}$

Adrenal gland is a complex poly functional organ that secretes hormones required for maintenance of life. According to Cannon (1968b) adrenal glands are emergency glands as they are called into action to control sudden explosive outbursts of energy. Adrenal gland is frequently affected by structural derangement of its components, which in turn leads to circulatory collapse or hypoglycemic coma and death. ${ }^{3}$

It is a composite of two neuroendocrine organs the cortex which is mesodermal and medulla that is neurectodermal (neural crest) in development. Structurally and functionally, it consists of two distinct zones the cortex and the medulla. Despite their organization into a single gland, the medulla and cortex are functionally different endocrine organs and have different embryological origins. The utility, if any, of having them together in one discrete organ is not obvious. In some species, amphibians and certain fishes, two separate organs are found. 4,5

The coelomic epithelium close to the root of dorsal mesentery opposite the sixth to twelfth thoracic segment proliferates into the underlying mesenchyme and give rise to the cortex of suprarenal gland and the migrating sympathochromaffin cells from the neural crest give rise to the medulla. The gland later invested by a capsule, which is derived from mesonephros. Foetal suprarenal gland is characterized by a thin peripheral permanent cortex and thicker deeper foetal cortex. ${ }^{6}$

The human foetal suprarenal gland is structurally quite different from its adult counterpart. The human foetal suprarenal gland most distinctive feature, the histologically unique foetal zone, was described first by Elliott and Armour in 1911. After the first trimester, the centrally located foetal zone accounts for most of the foetal adrenal mass. The outer zone of the foetal suprarenal is called the "definitive zone or neo cortex"; this zone likely gives rise to the adult adrenal glomerulosa. A third zone called "transitional zone", lies just between the neocortex and foetal zone and is believed to develop into the zona fasciculata. ${ }^{7,8}$

We therefore consider it of value to study the morphological and developmental anatomy and histogenesis of developing human foetal suprarenal glands at different gestational ages.

\section{AIMS AND OBJECTIVES}

The present study on developmental histology of human foetal adrenal glands of different gestational ages was conducted as a sample study with the following aims and objectives:

- To study temporal relations in histogenesis of adrenals.

- To study the suprarenal gland histology at different age groups.

\section{MATERIALS AND METHODS}

This work was conducted in the department of Anatomy, S.V.Medical College, Tirupati with the co-operation of Department of Anatomy, SVIMS, Departments of Obstetrics and Gynaecology, S.V. Medical College Tirupati. Institutional ethics committee approved collection of embryonic tissue after obtaining informed consent from patients and or kith and kin of the diseased. Materials studied include spontaneously aborted/dead foetuses of different periods of intra uterine life.

A total of 28 formalin preserved dead embryos and foetuses obtained from the Government Maternity Hospital, Tirupati with relevant obstetric records were preserved in department of Anatomy, S.V.Medical College, Tirupati and the same were utilized for this study.

The embryos and foetuses both sexes are of 05-40 weeks gestational age. Among these foetuses eight were embryos of less than 10 weeks gestational age, which were later subjected to serial sectioning. Others were aged between 12 to 40 weeks gestational age.

The Crown-rump length (CRL), Crown-heel length (CHL) and weight of the foetuses were recorded immediately after they are transported to the department of Anatomy. Subsequently the foetuses were preserved by injection of $10 \%$ formalin solution in to the pleural, peritoneal and cranial cavities.

\section{Collection of the specimen}

Two approaches for collecting suprarenal glands viz., posterior approach and an anterior approach were described in the literature. ${ }^{9}$

\section{Posterior approach}

In posterior approach the incision is given over the $11^{\text {th }}$ rib. The layers of thoracic wall in order from superficial to deep in this approach are skin, superficial fascia, Serratus posterior inferior muscle, External intercostal muscle, Internal intercostal muscle, Transversus thoracis muscle, endothoracic fascia, parietal pleura or peritoneum, 
pararenal fat, Gerota's fascia, perirenal fat and adrenal capsule. This approach described by Young (1936) offers the technical advantage of being extra peritoneal, extra pleural, and sub diaphragmatic. ${ }^{10}$

\section{Anterior approach}

has the advantage of simultaneous bilateral exploration when compared to posterior approach. ${ }^{11}$ A long curved transverse incision ("reversed smile") with its center point lying halfway between the umbilicus and the xiphisternum is placed on the anterior abdominal wall. A second vertical (midline or paramedian) incision is also given.

In the present study the anterior approach described by Cahill (1944) was followed. ${ }^{11}$

The anatomic stratifications in the incision line are:

1. Skin

2. Superficial fascia (Superficial fatty and deep membranous layers)

3. Deep fascia - thin areolar tissue covering the muscles

4. Muscles: External oblique, Internal oblique and Transversus

5. Fascia transversalis

6. Extraperitoneal fat

7. Parietal peritoneum

Histological appearance of suprarenals were studied under 4 groups. Group A of less than 12 weeks, group B of 13-24 weeks, group C of 25-36 weeks and group D of more than 36 weeks gestational age.

\section{Collection and processing of tissue for microscopy}

The collected specimens of adrenal glands were categorized according to their gestational age. Two specimens of one adrenal gland (right and left side) from each group were hemi sectioned coronally. The coronally sectioned tissues were subjected to routine tissue processing method for formalin preserved tissue. The paraffin block of tissue was cut into $6 \mu \mathrm{m}$ thick sections and stained with haematoxylin and eosin stains. The sections thus obtained were observed for the microscopic appearance under low power (10X), high power (40X) at different gestational ages. The histological sections were photographed by microphotography.

\section{RESULTS}

A total of 51 suprarenal glands bearing different gestational ages (12-more than 36 weeks) were processed for histological study. This includes the five small embryos of less than 10 weeks of gestational age that were serial embedded as a whole and were serially sectioned.
Histological observations of below 6 weeks to 36 weeks of gestational ages were described here under and depicted in Figures 1.1 to 1.12.

Histological observations of suprarenal glands in Group A (0-12 weeks gestational ages)

In the $10 \mathrm{~mm}$ CRL embryo that corresponds to Carnegie stage 14 and 4 to 5 weeks of post conception (Figure 1.1) the suprarenal glands were seen in the posterior abdominal wall, retroperitoneally at the level of lumbar vertebrae. Bilateral masses of cells are seen situated medial to the mesonephric bodies, having free surface covered by coelomic epithelium. These masses of cells are the anlage of foetal suprarenal, and can be readily distinguished from the surrounding tissues, the structure being that of embryonic connective tissue. The covering coelomic epithelium is of the same high columnar type as that covering the rest of the Wolffian ridge.

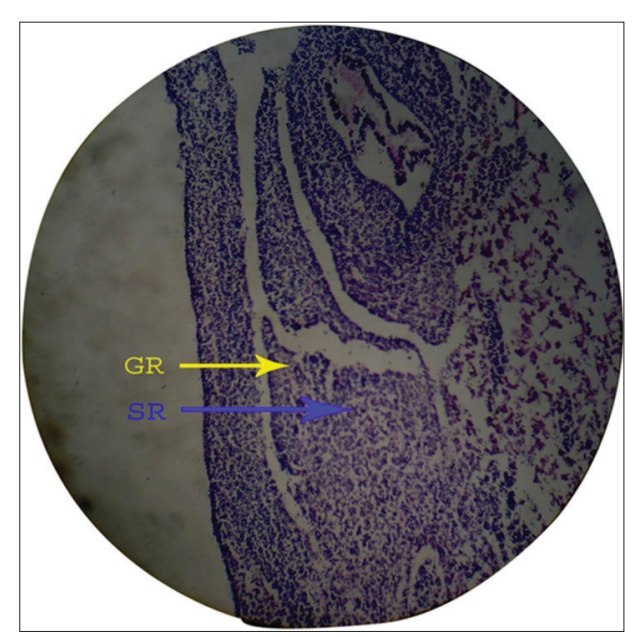

Figure 1.1: 4-5 Weeks Embryo CS 14 Stage sagital Section- Gonadal Ridge (GR) \& Suprarenal Gland (SR) in Lumbar Region (10X H \& E)

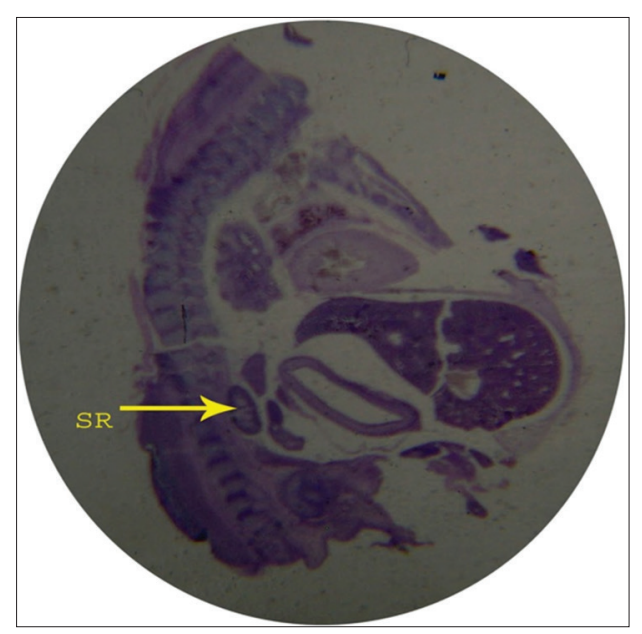

Figure 1.2: 6 Weeks Embryo $(12 \mathrm{~mm})$ CS 16 Stage Sagittal Section- (SR) Suprarenal Gland in Lumbar Region. (A) 10X (H \& E), (B) $10 \mathrm{X}(\mathrm{H} \& \mathrm{E})$ 
Sagittal section of the $12 \mathrm{~mm}$ CRL embryo that corresponds to Carnegie stage 15 and 6 weeks of post conception (Figure 1.2), presented definite suprarenal bodies on both sides of the aorta and pushing forwards in to the coelomic cavity medial to the mesonephros and developing genital gland. The cells of the coelomic epithelium immediately on either side of the root of mesentery are no longer columnar and are proliferating, forming a cap of cells 3 to 4 layers thick over developing gland. On the medial aspect of the gland migrating masses derived from the sympathetic origin are well seen, and form small groups of cells in the root of the mesentery, closely associated with the large blood vessels. These migrating bundles will be termed sympathochromaphil cells from now onwards, and few are already invading the organ.

In the $15 \mathrm{~mm}$ CRL sagittal section that corresponds to Carnegie stage 16 and 6-7 weeks of post conception (Figure 1.3), we obtained two embryos both shown more or less similar cellular architecture, even though one embryo is $15 \mathrm{~mm}$ in CRL, doesn't match with Carnegie stage 16 (Figure 1.4) as it is has 8 weeks of gestation according to records. The later exhibits definite paired suprarenal glands consisting of foetal cortex cells which are large, irregularly shaped and markedly eosinophilic, with large nuclei and well staining nucleoli. These cells are loosely arranged and the whole mass is very vascular. The migrating Sympathochromaphil masses are seen in root of the mesentery, and some bundles have already penetrated well into gland.

In the $17 \mathrm{~mm}$ CRL that corresponds to Carnegie stage 18 and 8-10 weeks of post conception (Figure 1.5), the suprarenal glands were easily recognized under microscope. Two glands are approximately of the same size. The sympatho-chromaphil element is represented by bilateral masses of cells similar to those seen in the sympathetic chains, situated between the aorta and suprarenal gland.

Histological observations of suprarenal glands in Group B (12-25 weeks gestational ages)

In this group we obtained 10 specimens (including right and left sides) gestational age ranges from 12-25 weeks and among these foetuses lowest age was 12 weeks and highest was 24 weeks. The general arrangement of the cells remains unchanged and the whole gland is very vascular, both small and large vessels having extremely thin walls. There is a well marked connective tissue capsule from which delicate strand run in to organ. Sympatho-chromaphil elements in the form of numerous bundles breaking off from these masses invade the glands chiefly on their medial aspect penetrated well into their substance. The cells pass into the bundles, which are surrounded by connective tissue, penetrating the capsule of the gland where they enter. In addition to this main invasion, bundles of cells passing in with the blood vessels also penetrate the gland on its lateral aspect. These masses of cells are seen as small bundles (Figures 1.6-1.8).

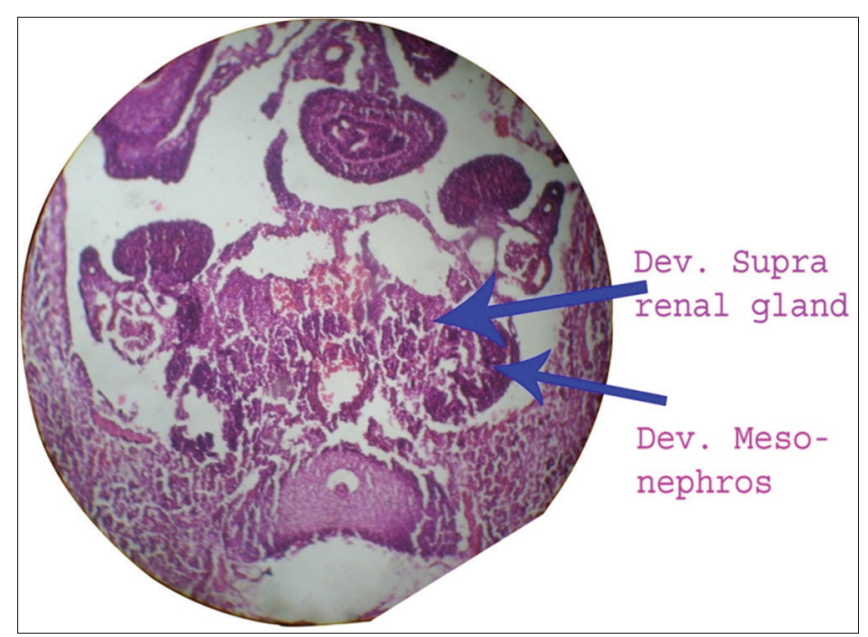

Figure 1.3: Horizontal Section through a $15 \mathrm{~mm}$ (16 weeks) embryo showing developing Suprarenal Gland (10X H \& E)

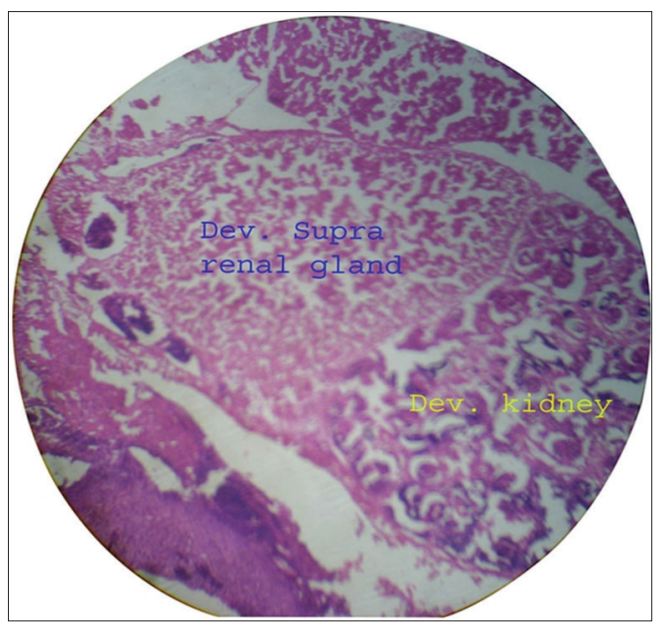

Figure 1.4: 15 mm Embryo 6-8 weeks coronal section (10X H \& E)

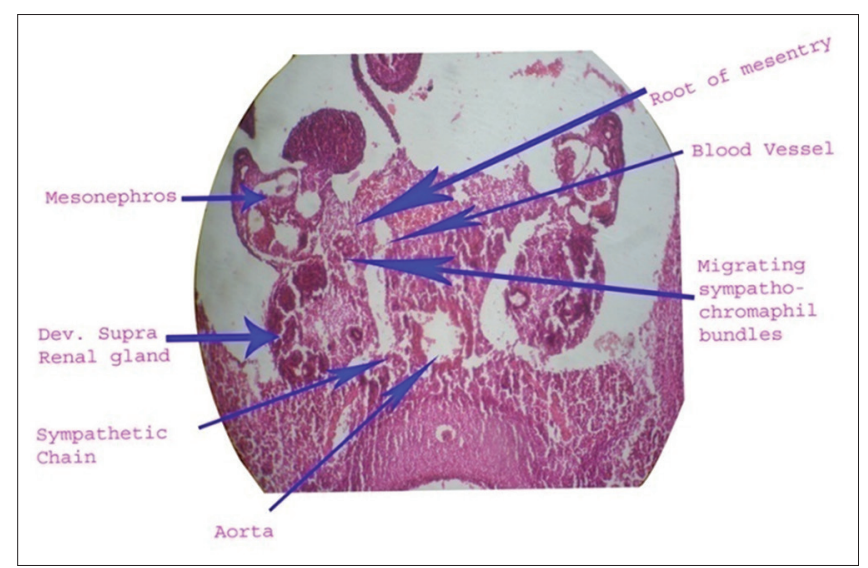

Figure 1.5: Horizontal section through Suprarenal Region of $17 \mathrm{~mm}$ (8-10 weeks) embryo (10X H \& E) 
Histological observations of suprarenal glands in Group C (25-36 weeks gestational ages)

In this group we studied 22 specimens (including right and left sides) gestational age ranging from 25 to 36 weeks and among these foetuses lowest age group was 25 and highest was 35 weeks. They showed same cellular architecture with marked fibrous capsule, and there is observable demarcation of foetal cortex to medullary zone and the central part is occupied by large thin walled spaces filled with blood around which penetrating bundles are collecting. In this group, medullary immigration was very active, the invading masses consisting for the greater part of the larger cells.

Histological observations of suprarenal glands in Group D (36 and more than 36 weeks gestational ages)

In this group we studied 14 specimens (including right and left sides) of gestational age ranging from 36 and more than 36 weeks and among these foetuses lowest and highest age were 36 weeks. In these last week nearly full term glands the foetal cortex increases gradually in thickness, immigration of cells is still well marked, the penetrating

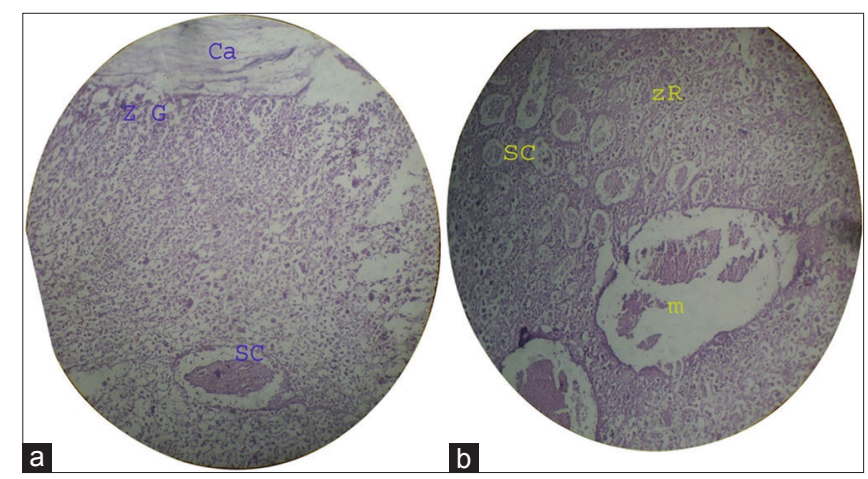

Figure 1.6: (a) 12 weeks foetal suprarenal gland showing huge amount of Capsule (Ca), with Peri- adrenal tissue and vesicular arrangement of cells of Zona Glomerulosa (ZG), Medullary region shows invagination of sympatho-Chromaphil bundles (SC) (10X H \& E) (b) In the same foetus suprarenal gland showing Zona Reticularis (zR), sympathoChromaphil bundles (SC) and medulla (m) were shown (40X H \& E)

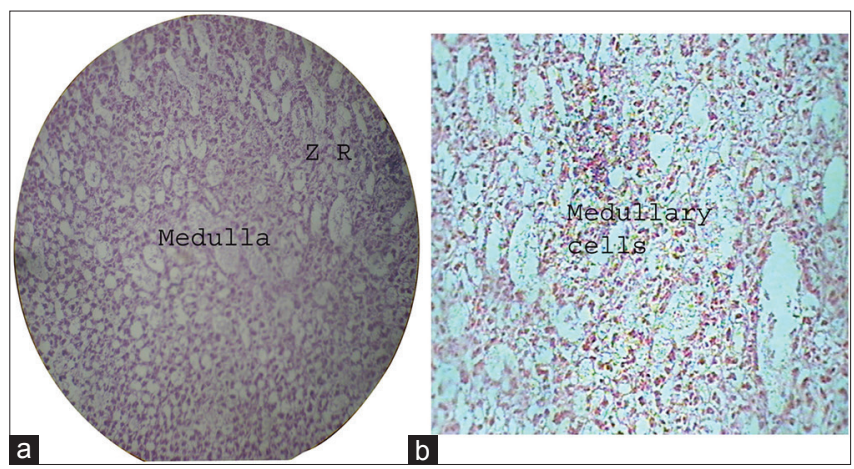

Figure 1.7: 18 Weeks foetus showing rosette arrangement of invading cells within the suprarenal gland (a. 10X H \& E), (b. 20X H \& E) bundles consisting at that time almost entirely of the larger type of cell. Nucleated cells, apparently erythroblasts are frequently seen in capillaries and visible sinuses. The arrangement of the parenchymal cells differs at different levels between the capsule and medulla. Three different arrangements are seen. Immediately below the capsule the parenchymal cells are grouped into little irregular clusters (Zona glomerulosa) with capillaries between the clusters. Beneath this is a thick layer (zona fasciculate) in which the cells are arranged in fairly straight cords which run at right angles to surface and have straight capillaries between them. Between the fasciculata and medulla is a relatively thin layer called the zona reticularis (Figures 1.9-1.12).

\section{DISCUSSION}

\section{Group A (0-12 weeks)}

For the histological studies, we used five embryos, which were serially sectioned. Among the five embryos, two were $15 \mathrm{~mm}$ CRL and others were $10 \mathrm{~mm}, 12 \mathrm{~mm}$ and $17 \mathrm{~mm} \mathrm{CRL}$

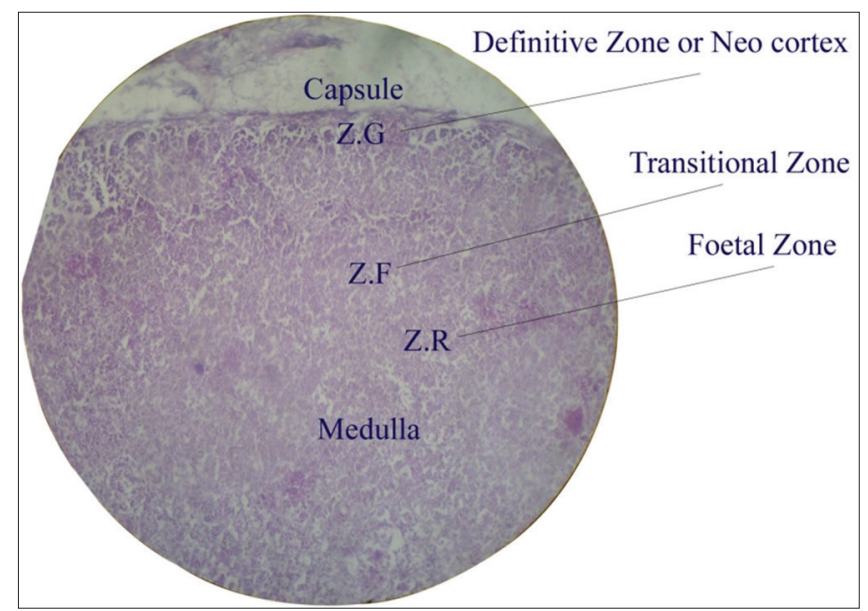

Figure 1.8: 22 weeks gland showing Different Zones (10X H \& E)

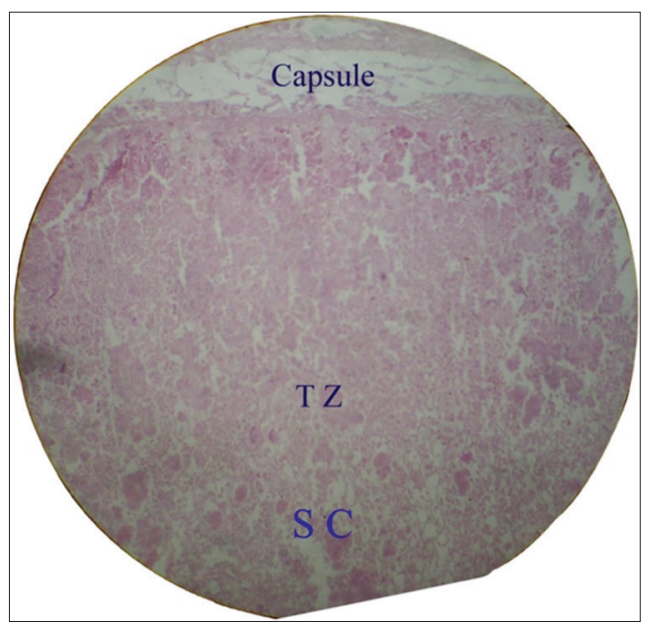

Figure 1.9: 28 weeks gland showing Transitional Zone (T Z) with Capsule and Sympatho-Chromaphil tissue (S C) (10X H \& E) 


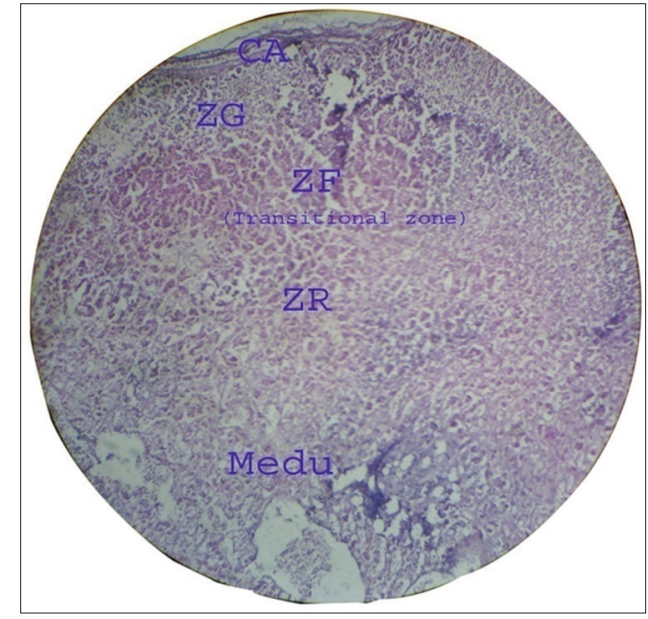

Figure 1.10: 30 weeks gland showing zonation and Medullary tissue and well marked transitional zone (10X H \& E)

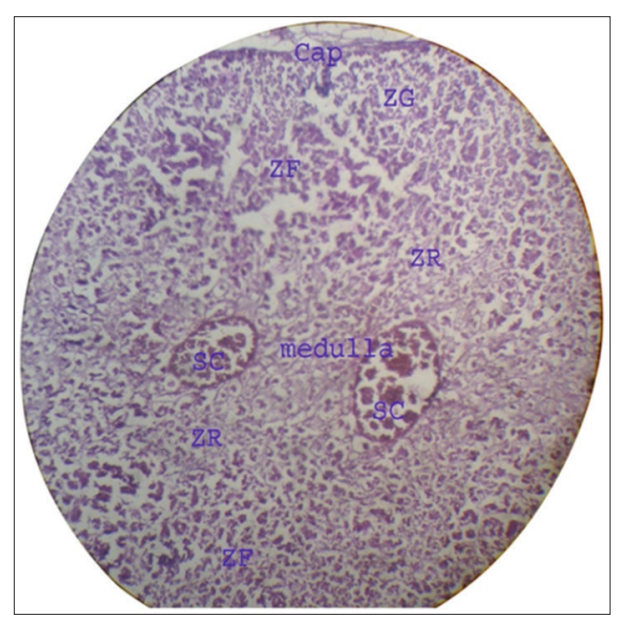

Figure 1.11: 33 weeks gland showing Scattered island of Primitive Sympathicoblasts, some of which reside in inner foetal cortex (10X H \& E)

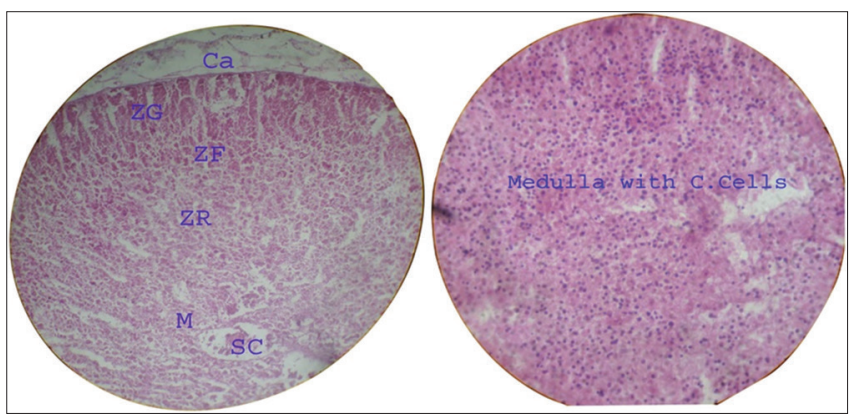

Figure 1.12: 36 weeks gland showing adult pattern of cellular architecture (10X H \& E)

embryos (Figure 1.1 to 1.5). Regarding the developmental origin of the true and foetal cortex the present study is in agreement with those reported earlier in the literature. Cap of proliferating coelomic epithelial cells were observed in the $10 \mathrm{~mm}$ embryo that represent true anlage of the true capsule (Figure 1.1). These cells appear to be migrating postero-laterally between the developing mesonephros and suprarenal mass that was differentiated in $12 \mathrm{~mm}$ embryo (Figure 1.2). On the medial aspect there is a procession of Sympatho-chromaphil bundles but without capsule (Figures 1.3 - 1.5). These observations are in agreement with earlier literature. ${ }^{1,4}$

With regards to medulla of the gland our findings agree with the observations of others, namely Vincent S, Curtis FR 1927, Sangma GTN 2008 that it is derived from the sympathetic anlage. ${ }^{12,6}$

\section{Group B (12-25 weeks)}

In this group we studied 10 suprarenal glands of 12 , 18, 22 and two 24 weeks of gestation (Figure 1.6 to 1.8) showed the general arrangement of the cells remaining unchanged and the whole gland was very vascular, both small and large vessels having extremely thin walls. By now there is a well marked connective tissue capsule from which delicate strand run in to organ. ${ }^{6}$ Sympathochromaphil elements in the form of numerous bundles breaking off from these masses invade the glands chiefly on their medial aspect, and penetrate well into their substance. The cells pass in bundles surrounded by connective tissue, penetrating the capsule of the gland where they enter. In addition to this main invasion, bundles of cells pass in with the blood vessels and also penetrate the gland on its lateral aspect. These masses of cells are well seen as small bundles. Above observation made by us were similar with the work of Cooper (1925), Knee and Hewer (1927). ${ }^{1,12}$

\section{Group C (25-36 weeks)}

In this group, we studied 22 suprarenal glands of 25,28 , 29,30,31,33 and two 33 and three 35 weeks of gestation (Figure 1.9 to 1.11). They showed same cellular architecture, well defined fibrous capsule, observable demarcation of foetal cortex to medullary zone with the central part occupied by large thin walled spaces filled with blood around which penetrating bundles were found collecting. In this group, medullary immigration was very active, the invading masses consisting for the greater part of the larger cells. These observations also agreed with the studies of Cooper (1925). $5,12,13,14$

\section{Group D (36 and more than 36 weeks)}

In this group, we studied 14 suprarenal glands of 36 weeks of gestation (Figure 1.12). The foetal cortex increased gradually in thickness. Immigration of cells is still well marked, the penetrating bundles consisting at that time almost entirely of the larger type of cell. Nucleated cells, apparently erythroblasts were frequently seen in capillaries and visible sinuses. These observations are in agreement with those reported by Cooper (1925)..$^{12,13}$ 


\section{CONCLUSIONS}

A total of 5 embryos and 23 human foetuses were studied for age related histological features of suprarenal glands. Five foetuses of $>12$ weeks gestational age were subjected to serial sections and the same were observed for location, and early histogenesis of suprarenal glands.

Histological observation of the all the specimens observed in the present study are in agreement with those reported in the literature excepting that they appeared earlier in the present study than that reported in the literature. Capsule of suprarenal gland appeared at 12 weeks, sympathochromaffin bundles appeared before 6 weeks and zonation of cortex was observed at 8 weeks in the present study when compared to the time of appearance reported in the literature as 14 weeks, after 6 weeks and after 12 weeks respectively in the literature.

It is concluded from the present results that histological studies reveal that primary cortical proliferation began by the $4^{\text {th }}$ week of gestation from coelomic epithelial cells between the root of the dorsal mesentery and urogenital ridge. Suprarenal fibrous capsule and zonation of foetal cortex were observed by 12 weeks of gestation.

Blood vessels were appearing at the age of 8 weeks and gradually increased in their network as sinusoids at the age of 22 weeks.

The immigration of the Sympatho-chromaphil masses began at about 6 weeks of development, most active between the $12^{\text {th }}$ to $22^{\text {nd }}$ weeks, and ceases about full time.

As the observations were on a small population during the limited period of study the present work needs to be extended on a larger population of different age groups. For histological interpretation use of special stains and markers are needed for establishing a base line data that can be used for interpreting normal developmental pattern and for explaining developmental abnormalities.

\section{ACKNOWLEDGEMENT}

Special thanks the Dr.R. Sekhar, Dr.V.Usha Rani, Dr.Vasudeva Reddy, Professor, Dept. of Anatomy, Sri Venkateswara Medical College, Tirupathi, Andhra Pradesh, India.

\section{REFERENCES}

1. Crowder R. The development of the adrenal gland in man, with special reference to origin and ultimate location of cell types and evidence in favor of the "cell migration" theory. Contrib. Embryol Carnegie Inst 1957; 36:193.

2. Nowak D, Goralczyk K, Zurada A and Gielecki J. Morphometrical analysis of the human suprarenal gland between the $4^{\text {th }}$ and $7^{\text {th }}$ months of gestation. Annals of Anatomy- Anatomischer Anzeinger 2007; 189(6):575-582.

3. Dobbie JW and Symington T. The human adrenal gland with special reference to the vasculature. Journal of Endocrinology 1966; 34(4):479-NP.

4. Douglas E.Kelly, Richard L wood, Allen C Enders. Baileys text book of histology; $18^{\text {th }}$ Edition 1983. Pp. 517-523.

5. Zuckerkandl E. The development of the chromaffin organs and of the suprarenal glands. P. 157. In KeibelF and Mall EP (eds): Manual of human Embryology. JB Lippincott, Philadelphia, 1912.

6. Sangma GTN, Ibochouba $Y$ and Damayanti N. Development and maturation of suprarenal glands in Human fetuses. J Anat Soc India 2008;57(1):1-7.

7. Perlman RL and Chalfie M. Catecholamine release from the adrenal medulla. Clinics in endocrinology and metabolism 1977;6(3):551-576.

8. David N. Orth, William J. Kovacs, and C. Rowan DeBold, Williams. Text books of Endocrinology $8^{\text {th }}$ Edi. Wilson \& Foster. 2004. Pp. 248-252.

9. Franksson $\mathrm{C}$ and Hellstrom J. Bilateral Adrenalectomy, with particular reference to operative technique. Acta Chir Scand 1956;111:54.

10. Young HH. A Technique for Simultaneous Exposure and Operation on the Adrenals, Surg Gynec and Obst 1936; 63:179-188.

11. Cahill GF. Hormonal tumors of the adrenals. Surgery 1944; 16:233.

12. Cooper ERA. The histology of the more important human endocrine organs at various ages. Oxford Press 1925.

13. Vincent $S$ and Curtis FR. A note on the teleostean adrenal bodies. Journal of Anatomy 1927; 62(Pt 1):110-115.

14. O'Rahilly R. The timing and sequence of events in the development of the human endocrine system during the embryonic period proper. Anatomy and embryology 1983; 166(3):439-451.

\footnotetext{
Authors Contribution:

RKB-Concept and design of the study, specimen processing, literature review, analyzing, Interpretations manuscript preparation, critical revision of the manuscript, drafting of the manuscript; SDV- Concept and design of the study, critical revision of manuscript and review of the study.

Work attributed to:

Department of Anatomy, Sri Venkateswara Institute of Medical Sciences, SVIMS University, Tirupathi, Andhra Pradesh.

Orcid ID:

Dr. Ravindra Kumar Boddeti - (D) https://orcid.org/0000-0003-0569-1472

Dr. Subhadra Devi Velichety - iD https://orcid.org/0000-0002-3995-8449

Source of Support: None, Conflict of Interest: None.
} 